\title{
Chlorins e6 loaded silica nanoparticles coated with gastric cancer cell membrane for tumor specific photodynamic therapy of gastric cancer
}

This article was published in the following Dove Press journal: International Journal of Nanomedicine

\author{
Jiaxing Yang' \\ Yongliang Teng ${ }^{2}$ \\ $\mathrm{Yu} \mathrm{Fu}{ }^{3}$ \\ Chunyu Zhang ${ }^{3}$ \\ 'Department of Gastrointestinal Surgery, \\ The First Hospital of Jilin University, \\ Changchun, Jilin, People's Republic of \\ China; ${ }^{2}$ Department of Pathology, The \\ First Hospital of Jilin University, \\ Changchun, Jilin, People's Republic of \\ China; ${ }^{3}$ Department of Radiology, The \\ First Hospital of Jilin University, \\ Changchun, Jilin, People's Republic of \\ China
}

Background: Photodynamic therapy (PDT) is widely recognized as a promising way to cure cancer. However, the limited tumor homing property of currently available drug delivery systems (DDSs) is the bottleneck for the delivery of photodynamic agents.

Purpose: In our study, we decorated silica nanoparticles (SLN) with cell membrane (CM) derived from SGC7901 cells to construct carrier (CM/SLN) which was able to to specifically target the homogenous SGC7901 cells.

Materials and methods: Furthermore, the decent drug loading capability of CM/SLN was adopted to load photodynamic agent chlorins e6 (Ce6) to finally construct aDDS suitable for tumor-targeted PDT of gastric cancer.

Results: The experimental results suggested that CM/SLN/Ce6 was nano-sized particles with good dispersion and stability in physiological conditions. Moreover, due to the modification of CM,CM/SLN/Ce6 could specifically target the homogenous SGC7901 cells both in vitro and in vivo. Most importantly, further in vivo results demonstrated that the CM/SLN/ Ce6 showed a better anticancer outcome compared to SLN/Ce6.

Conclusion: $\mathrm{CM} / \mathrm{SLN} / \mathrm{Ce} 6$ might be a promising platform for effective tumor targeted PDT of gastric cancer.

Keywords: cell membrane, silica nanoparticles, chlorins e6, photodynamic therapy, gastric cancer

\section{Introduction}

In the past decades, the nanoparticle-based drug delivery systems (DDS) have widely applied in cancer therapy. Compared to free drugs, they showed elevated bioavailability and decreased side effects which holds great potential for clinical applications. ${ }^{1-4}$ Herein, previous reports have constructed different kinds of nanoparticles based DDSs using materials from organic to inorganic ones, ${ }^{5-8}$ among which silica nanoparticles (SLN) with versatile merits is emerging as a potential candidate. 9,10 Therefore, many DDSs have been developed using SLN as the skeleton that achieved benificial outcomes both in vitro and in vivo. ${ }^{11-13}$

However, the targeted delivery is still unsatisfied in many currently available DDSs which requires further improvement. ${ }^{14}$ To address this dilemma, the modification of DDSs with targeting ligands is one of the mostadopted ways. $^{6,15}$ In the past decades, targeting ligands varying from small molecules to monoclonal antibodies, have been successfully integrated into DDS to improve its tumor-targeting capabilities. ${ }^{16-18}$ However, the ectogenic nature of
Correspondence: Chunyu Zhang Department of Radiology, The First Hospital of Jilin University, Changchun, Jilin I3002I, People's Republic of China Email zhangchunyu_vic@I63.com 
these ligands might induce some unexpected side effects, such as immunoreaction and cytotoxicity.

Photodynamic therapy (PDT) is a rapidly developing technic that employ photodynamic agents to transfer NIR light into highly ROSto exert cytotoxic on cells. ${ }^{19}$ As a noninvasive and harmless approach, PDT is well recognized as a preferable measure for cancer therapy. ${ }^{20}$ Chlorins e6 (Ce6) is known as a commonly adopted PDT agent, both in experimental and clinical applications, which has irreplaceable advantages over other agents. $^{21,22}$ However, Ce6 was also found to have some limitations, including irreversible degradation, poor solubility as well as rapid photobleaching, which call for the aid of multifunctional DDSs. ${ }^{23}$

Cellular membranes (CM) were reported to be involved in many vital biological processes, which mainly ascribed to the proteins in the phospholipid bilayer. ${ }^{24}$ In addition, the $\mathrm{CM}$ also shows high biocompatibility and inherits the merits of the source cells. $^{25}$ As a result, CM has been used to construct tumor-targeting nanoparticles for the treatment of various carcinomas, ${ }^{26}$ in which CM have been shown to endow the modified nanoparticles with great tumor homing ability while at the same time reduce the undesired side effects of drugs in a facile and flexible way. ${ }^{27,28}$

With the aim of combiningCM and Ce6 in one DDS with tumor targetability for advanced PDT, amine SLN with positive surface charge was firstly synthesized and loaded with Ce6 (SLN/Ce6). Subsequently, the SLN/Ce6 was coated with negatively charged CM (derived from SGC7901 cells) to fabricate a tumor targeted platform (CM/SLN/Ce6). Compared with other currently available PDT DDSs, it was expected that CM modification coulddirect the CM/SLN/Ce6 to the homogenous SGC7901 cell line to increase its tumor targeted accumulation. Afterwards, the released $\mathrm{Ce} 6$ molecule within cancer cells could achieve sufficient PDT of gastric cancer upon the irritation of external NIR light.

\section{Materials and methods}

\section{Materials}

Indocyanine green (ICG), Ce6, Triton X-100, $\mathrm{N}$-(2-aminoethyl)-3-aminopropyltrimethoxysilane (AEAPS), tetraethyl orthosilicate (TEOS), 2',7'-dichlorodihydrofluorescein diacetate (DCFH-DA), 1,3-diphenylisobenzofuran (DPBF) and methylthiazoletetrazolium (MTT) were obtained from Sigma-Aldrich (St Louis, MO, USA). Chemicals and regents otherwise stated were purchased from Aladdin Co., Ltd (Shanghai, China) andanalytically pure.

\section{Cell culture and animal model}

The SGC7901 cell line (human gastric carcinoma) was purchased from American Type Culture Collection (ATCC, Manassas, VA, USA) and cultured using standard protocol according to previous report. ${ }^{29}$

Female Balb/c nude mice (6 8 week) were obtained from Model Animal Research Center of Nanjing University (Nanjing, China) and the SGC7901 tumor xenograft model was established as previous reported. ${ }^{29}$ All animal procedures strictly followed the NIH guidelines for the care and protection of laboratory animals and were confirmed by the Ethics Committee of the First Hospital of Jilin University.

\section{Preparation of CM/SLN/Ce6}

The amine SLN was prepared using a water-in-oil microemulsion according to a previousreport. ${ }^{30}$ In brief, the water-in-oil microemulsion was firstly prepared and stirred properly to obtain transparent solution. Afterwards, TEOS, AEAPS and $\mathrm{NH}_{4} \mathrm{OH}$ were successively added into the microemulsion to initiate the reaction. After being stirred at room temperature for 24 hours, the solution was charged with additional ethanol, followed by centrifugation (3,000 rpm, 10 minutes, CR21, Hitachi Ltd, Tokyo, Japan) to collect amine SLN as precipitate. In order to obtain Ce6 loaded SLN (SLN/Ce6), the collected SLN was suspended in the proper amount $(5 \mathrm{~mL})$ of DMSO in which Ce6 was dissolved to achieve drug concentration of $5 \mathrm{mg} / \mathrm{mL}$. The solution was agi.tated under room temperature for 24 hours and then centrifuged to collect SLN/ Ce6 as mentioned above. The product was washed with ethanol several times and stored at $4{ }^{\circ} \mathrm{C}$ until used.

The isolation of CM from SGC7901 cells was performed according to a previous report. ${ }^{31}$ In brief, the SGC7901 cells were seeded into culture dish $(200 \mathrm{~mm})$ and allowed to growto $90 \%$ confluence. Afterwards, cells were washed with PBS, detached with tyrisin and concentrated in $3 \mathrm{~mL}$ of dispersion buffer. The cells were centrifuged at 1,000 rpm for 5 minutes, and the collected pellet was homogenized in $1 \mathrm{~mL}$ of extracting buffer. Afterwards, the mixture was further centrifuged at $10,000 \mathrm{rpm}$ for 10 minutes. The supernatant was again ultracentrifuged (100,000 rpm for 60 minutes). The CM pellet was resuspended and stored. The protein in CM was quantified by BCA kit (Beyotime, Shanghai, China).

The coating of CM onto SLN/Ce6 was performed according to previously reported method. ${ }^{32}$ Briefly, $250 \mu \mathrm{L}$ of SLN/ 
Ce6 (nanoparticle concentration: $1 \mathrm{mg} / \mathrm{mL}$ ) was mixed with different volume SGC7901 CM solution under vortex. Afterwards, the mixture was subjected to probetype sonication (100 W, 5 minutes). The mixture was further centrifuged (10,000 rpm, 10 minutes) to collect CM/SLN/Ce6.

The drug loading content (DLC) of CM/SLN/Ce6 was determined by immersingthe prepared nanoparticles in methanol for 48 hours. After being centrifuged at $10,000 \mathrm{rpm}$ for 30 minutes, the content of $\mathrm{Ce} 6$ in the supernatant was determined using HPLCwith the method described as follows: Thermo-Fisher UltiMate ${ }^{\mathrm{TM}} 3000$ (Thermo Fisher Scientific, Waltham, MA, USA) equipped with Phenomenex- $\mathrm{C}_{18}$ column $(250 \mathrm{~mm} \times 4.6 \mathrm{~mm}, 5 \mu \mathrm{m})$, mobile phase consisted of mixed methanol- ammonium acetate aqueous buffer $(0.05 \mathrm{M}, \mathrm{pH} 5.5$ and was adjusted to 3.0 by acetic acid); the wavelength was set at $405 \mathrm{~nm}$, flow rate was $1 \mathrm{~mL} /$ minute and temperature was $30^{\circ} \mathrm{C}$

\section{Characterization}

The size distribution of CM/SLN/Ce6 was measured by size analyzer (ZS90, Malvern). The morphology was observed using a transmission electron microscope (TEM, JEM-2100, JEOL, Japan).

Total protein from the CM was extracted using RIPA lysis buffer (Thermo Fisher) following the manufacturer's instructions, followed by concentration quantification using the $\mathrm{BCA}$ kit. Afterwards the Western blot analysis was performed strictly according to a previous report. ${ }^{33}$ Briefly, samples were resolved gel electrophoresis, transferred onto PVDF membrane, stained with corresponding first antibodies and followed by incubation with IRDyeR680CW-labeled second antibody. Finally, the blots were observed using a densitometer (E-Gel Imager, Thermo-Fisher). Each sample was repeated in triplicate and beta-actin was employed as control.

\section{Stability assay}

The CM/SLN/Ce6 was diluted using PBS and mouse plasma, respectively. The changes of particle size was recorded for 48 hours. In addition, the fluorescence intensity of $\mathrm{CM} / \mathrm{SLN} / \mathrm{Ce} 6$ against $\mathrm{Ce} 6$ in solution was also monitored using fluorescence spectrophotometer (F-7000, Hitachi) for 6 days. ${ }^{34}$

\section{In vitro release and in vitro ROS generation}

The $\mathrm{Ce} 6$ release from $\mathrm{CM} / \mathrm{SLN} / \mathrm{Ce} 6$ was studied using previous reported protocal. $^{29}$ In brief, CM/SLN/Ce6 was loaded into a dialysis bag, immersed in $25 \mathrm{~mL}$ of PBS (pH of 7.4 and 5.5), and fixed in a thermostatic shaker (SY2230; Crystal Technology, Texas, USA). At different time points, aliquot buffer was withdrawn to determine the drug concentration using HPLC as mentioned above.

The DPBF was adopted to test the generation of singlet oxygen as reported in a previous article. ${ }^{35}$ In brief, $20 \mu \mathrm{L}$ of DPBF (10 mM in DMSO) solution was added to CM/SLN/ Ce6 under gentle agitation. The mixture was irradiated with a $680 \mathrm{~nm}$ laser bean $\left(1 \mathrm{~W} / \mathrm{cm}^{2}\right)$. The time or concentration dependent variations of absorption peak at $418 \mathrm{~nm}$ of DPBF were measured by UV spectrophotometer.

\section{Cytotoxicity assay}

Drug free nanoparticles $(5-100 \mu \mathrm{g} / \mathrm{mL})$ or CM/SLN/Ce6 (Ce6 concentration, 0.25-5 $\mu \mathrm{g} / \mathrm{mL}$ ) were cultured with SGC7901 cells for 48 hours and the subjected to a standard MTT assay. The irradiation wavelength was $680 \mathrm{~nm}$ at $1 \mathrm{~W} / \mathrm{cm}^{2}$ for 5 minutes in all Ce6 containing groups.

\section{Cellular uptake and ROS detection}

In order to observe the internalization profile of $\mathrm{CM}$ / SLN/Ce6 in SGC7901 cells. Cells were firstly seeded in 6 -well plates with $70 \%$ confluence and then cultured with free $\mathrm{Ce} 6$, SLN/Ce6 and CM/SLN/Ce6 at the Ce6 concentration of $2 \mu \mathrm{g} / \mathrm{mL}$. For competitive binding assay, cells were pretreated with excessive CM for 2 hours prior to the addition of formulations. At prearranged time intervals, cells were rinsed with fresh HBSS and then subjected to confocal laser scanning microscope (CLSM, BX61W1-FV1000, Olympus Corporation, Tokyo, Japan) observation. In addtion, cells were harvested and analyzed by flow cytometer (FCM, Attune NxT, Thermo Fisher).

DCFH-DA is a cell permeable derivative of DCF which remains nonfluorescent during endocytosis. However, the oxidization product of DCFH-DA can exert greatly enhanced fluorescent intensity upon exposure to ROS. To detect the intracellular ROS levels, SGC7901 cells were firstly incubated with different formulations for 4 hours (Ce6 concentration: $0.1 \mathrm{mg} / \mathrm{mL}$ ). Afterwards, DCFH-DA $(25 \mathrm{mM})$ was added to incubated with cells at $37^{\circ} \mathrm{C}$ in the dark for 30 minutes. Thereafter, cells were exposed to $680 \mathrm{~nm}$ irradiation (1 $\mathrm{W} / \mathrm{cm}^{2}, 5$ minutes), followed by CLSM observation as mentioned above. 


\section{In vivo tumor targeting of CM/SLN}

ICG was loaded into SLN and CM/SLN in place of Ce6. Mice bearing SGC7901 tumor were intravenously administered with SLN/ICG and CM/SLN/ICG (20 $\mu \mathrm{g} /$ mouse of ICG) and the in vivo distribution of ICG signal at 6 and 12 hours was recorded by imaging system (ZEWTON 7.0, Vilber, Marnela-Vallée, France). Moreover, the mice were sacrificed at 48 hours post injection and the fluorescence intensity of the both formulations in tumor tissues and major organs was assessed using the same system.

\section{In vivo antitumor assay}

Mice were randomly divided into 4 groups $(\mathrm{N}=6)$ and administered with saline (as control), free Ce6, SLN/Ce6 and $\mathrm{CM} / \mathrm{SLN} / \mathrm{Ce} 6(5 \mathrm{mg} / \mathrm{kg} \mathrm{Ce}$ ). The irradiation wavelength was $680 \mathrm{~nm}$ at $1 \mathrm{~W} / \mathrm{cm}^{2}$ for 5 minutes in all Ce6 containing groups. The administration and other protocols follow the previous report. ${ }^{36}$

\section{Statistical analysis}

Statistical analysis was performed according to a previous report. ${ }^{37}$ In brief, experiments were conducted in a parallel manner and data were presented as mean \pm SD. Statistical significance was tested by two-tailed Student's $t$-test or one-way ANOVA $P<0.05$ or $P<0.01$ was considered as statistically significant.

\section{Results and discussion \\ Characterization of CM/SLN/Ce6}

In order to combine decent drug-loading capacity and biocompatibility in one DDS, SLN as a widely adopted biomaterial was prepared by a previous reported water-in-oil microemulsion method and employed as the skeleton material. Afterwards, the optimal ratio between $\mathrm{CM}$ protein and SLN/Ce6 was explored by mixing SLN/Ce6 with different ratios of $\mathrm{CM}$ (SLN/Ce6 to CM protein, w/w). The particle size and zeta potential of different ratios were recorded. As indicated in Figure 1A, the SLN/Ce6 had a hydrodynamic size of $90.4 \pm 3.2 \mathrm{~nm}$ and a zeta potential of $+32.4 \pm 1.7 \mathrm{mV}$. Upon CM coating, the particle size of CM/SLN/Ce6 gradually increased with the increase of $\mathrm{CM}$ while the zeta potential decreased. This phenomenon suggested that SLN/Ce6 was successfully coated with CM. Interestingly, when the mass ratio excess 6 , both the particle size and zeta potential of $\mathrm{CM} / \mathrm{SLN} / \mathrm{Ce} 6$ reached a balance with additional $\mathrm{CM}$ resulted insignificant changes. In detail, the hydrodynamic diameter of $\mathrm{CM} / \mathrm{SLN} / \mathrm{Ce} 6$ reached 115.6 $\pm 1.6 \mathrm{~nm}$ while the surface charge of $\mathrm{CM} / \mathrm{SLN} / \mathrm{Ce} 6$ reversed to $-30.4 \pm 2.3 \mathrm{mV}$, which was similar to that of the $\mathrm{CM}$
A

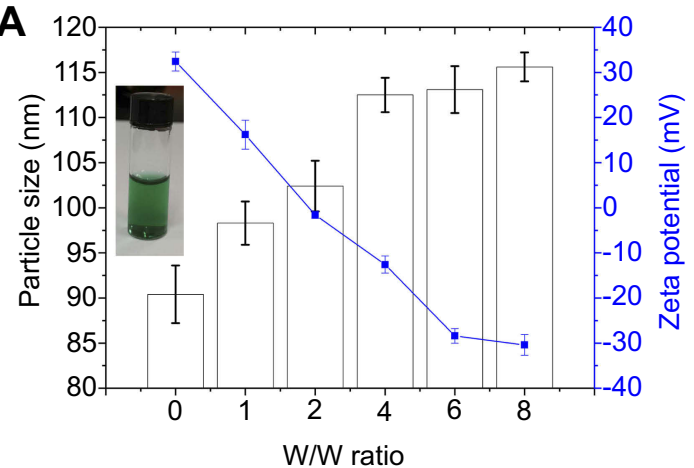

B SLN/Ce6

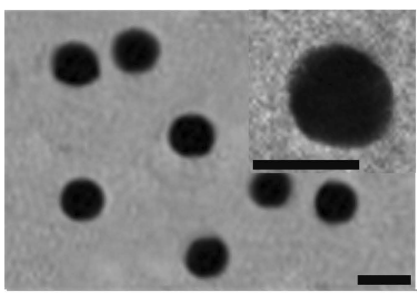

C

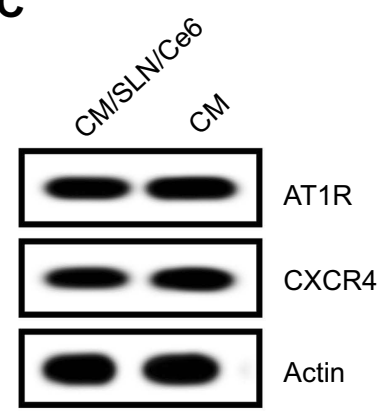

$\mathrm{CM} / \mathrm{SLN} / \mathrm{Ce} 6$
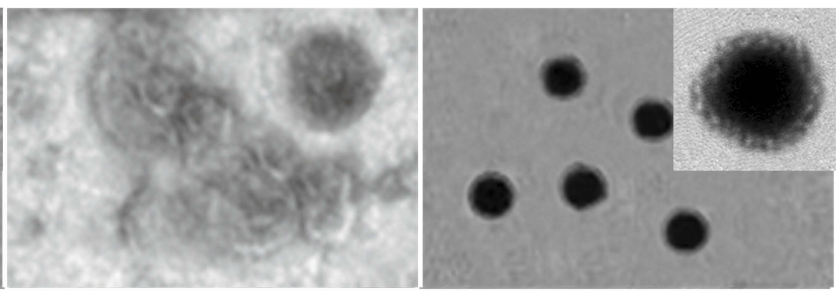

Figure I (A) Variation of particle size and zeta potential as a function of CM ratio (SLN/Ce6 to CM protein, w/w). Inset image was the optical image of CM/SLN/Ce6 at the optimal w/w ratio, which provides direct evidence of successful Ce6 loading. (B) Western blot analysis of two proteins (ATIR and CXCR4) in CM and CM/SLN/Ce6. (C) TEM images of SLN/Ce6, CM and CM/SLN/Ce6. Scale bar: $100 \mathrm{~nm}$.

Abbreviations: Ce6, chlorins e6; CM, cellular membranes; TEM, transmission electron microsope; SLN, silica nanoparticles. 
vesicles $(-29.7 \pm 1.5 \mathrm{mV})$. These results indicated that $\mathrm{CM}$ was successfully coated onto SLN/Ce6 and the coating was saturated at the mass ratio of 6 . The negative surface charge of $\mathrm{CM} / \mathrm{SLN} / \mathrm{Ce} 6$ was expected to protect $\mathrm{CM} / \mathrm{SLN} / \mathrm{Ce} 6$ from nonspecific absorption of plasmatic proteins, which in turn enahnce the stability of $\mathrm{CM} / \mathrm{SLN} / \mathrm{Ce} 6$ during circulation. ${ }^{38}$

The formation of $\mathrm{CM} / \mathrm{SLN} / \mathrm{Ce} 6$ from $\mathrm{SLN} / \mathrm{Ce} 6$ was also verified using TEM. As shown in Figure 1B, TEM revealed that SLN/Ce6 exhibited uniform and spherical nanostructure with homogeneous diameter dispersion at around $90 \mathrm{~nm}$. CM vesicles showed irregular shape of particles with diameter ranging from nanometer to micrometer. The finally obtained hybrid CM/SLN/Ce6 was characterized by a spherical core-shell structure with a clearly observed lipid bilayer on the surface.

It has been demonstrated that proteins on CM are essentially responsible for the tumor-targeting capability of the modified nanoparticles. ${ }^{39}$ As a result, we selected two different membrane proteins (AT1R and CXCR4) and compared their expression pattern in both $\mathrm{CM}$ and $\mathrm{CM} / \mathrm{SLN}$ / Ce6 using Western blot assay. As displayed in Figure 1C, the expression levels of both proteins in both groups were comparative, which suggested that $\mathrm{CM} / \mathrm{SLN} / \mathrm{Ce} 6$ inherited the integrated protein profiles of CM without lost or degradation during the coating process. It was also considered as a solid evidence to verify the successful preparation of $\mathrm{CM} /$ SLN/Ce6.

The DLC of $\mathrm{Ce} 6$ in $\mathrm{CM} / \mathrm{SLN} / \mathrm{Ce} 6$ determined by HPLC was $15.34 \%$.

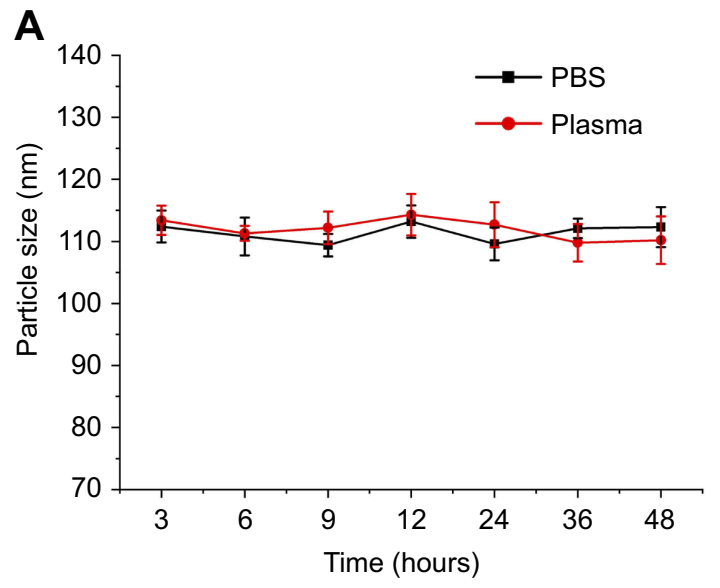

\section{Stability assay}

The stability of nanoparticles in physiological environment is generally considered as one of the basic requirements if a DDS is intended to successfully deliver the cargo. ${ }^{40}$ According to previous report, the in vivo fate of the DDS is stronglyrelated to its size. ${ }^{41}$ As a result, the capability to maintain its size without significant variation is vital for the DDS to bypass the complex extracellular barriers before arriving at the targeted sites. Herein, the size change of $\mathrm{CM} / \mathrm{SLN} / \mathrm{Ce} 6$ in PBS (pH 7.4) and mouse plasma was monitored for 48 hours to assess its colloidal stability. As shown in Figure $2 \mathrm{~A}$, the size of $\mathrm{CM} / \mathrm{SLN} / \mathrm{Ce} 6$ in both PBS and plasma at all tested time points showed insignificant changes, which suggested that $\mathrm{CM} / \mathrm{SLN} / \mathrm{Ce} 6$ might be able to withstand the irritation during circulation. Alternatively, Ce6 is prone to degrade upon light irritation in aqueous solution, which was reflected as photo bleaching. The DDS was expected to retard this process and increase the resistance of $\mathrm{Ce} 6$ to light irritation. As a proof of concept, the comparative fluorescence stability of $\mathrm{CM} / \mathrm{SLN} / \mathrm{Ce} 6$ and free Ce6 under sunlight irradiationfor 6 days was studied. As displayed in Figure 2B, free Ce6 showed immediate photo bleaching as the fluorescence intensity dropped dramatically to $61.5 \%$ only 1 day post irradiation, which continuously declined to $15.6 \%$ at the end of the test. In contrast, the fluorescentintensity in $\mathrm{CM} / \mathrm{SLN} / \mathrm{Ce} 6$ group showed much slowerdecrease as only $20.1 \%$ of the Ce6 suffered from photo bleaching at the end of the test. These results suggestedthat $\mathrm{CM} / \mathrm{SLN} / \mathrm{Ce} 6$ was capable of protecting the encapsulated $\mathrm{Ce} 6$ from light induced degradation, which is

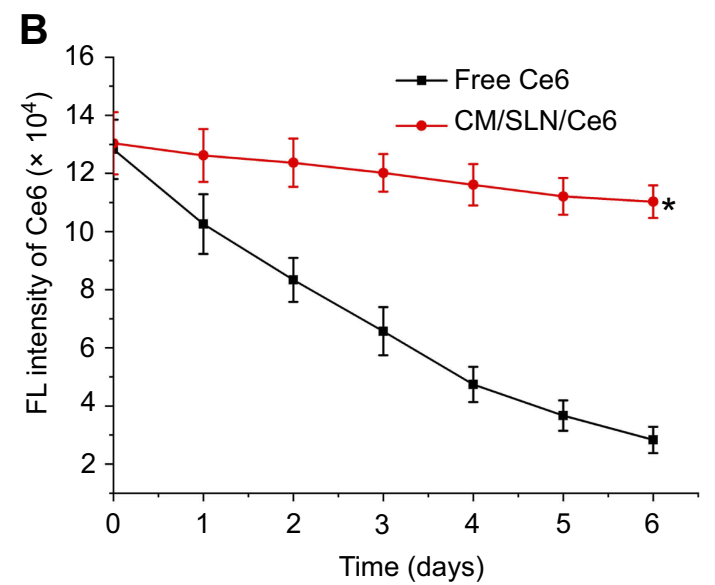

Figure 2 (A) Colloidal stability of CM/SLN/Ce6 in PBS ( $\mathrm{pH} 7.4$ ) and mouse plasma at $37^{\circ} \mathrm{C}$ for up to 48 hours. (B) Comparative fluorescentstability of CM/SLN/Ce6 and free Ce6.

Note: Data were shown as mean $\pm \mathrm{SD}(\mathrm{N}=3)$. ${ }^{*} \mathrm{p}<0.05$.

Abbreviations: FL, fluorescent; Ce6, chlorins e6; CM, cellular membranes; SLN, silica nanoparticles. 
favorable for CM/SLN to be developed as a stable system to satisfy further applications. ${ }^{42}$

\section{In vitro drug release and in vitro ROS generation}

The drug release pattern of $\mathrm{CM} / \mathrm{SLN} / \mathrm{Ce} 6$ was evaluated in two different $\mathrm{pH}$ conditions $(\mathrm{pH} 7.4$ and $\mathrm{pH} 4.5)$ to mimic the extracellular physiological environment and intracellular endolysosomal compartment. As illustrated in Figure 3A, after 120 hour of incubation, the accumulated drug release of $\mathrm{CM} / \mathrm{SLN} / \mathrm{Ce} 6$ at $\mathrm{pH} 7.4$ was relatively slower $(32.96 \%)$ compared to that of $\mathrm{pH} 5.5$ (74.61\%). It was inferred that the acidic environment could facilitate the drug release from $\mathrm{CM} / \mathrm{SLN} / \mathrm{Ce} 6$, which was beneficial for CM/SLN/Ce6 to serve as a responsive platform for advanced drug delivery.
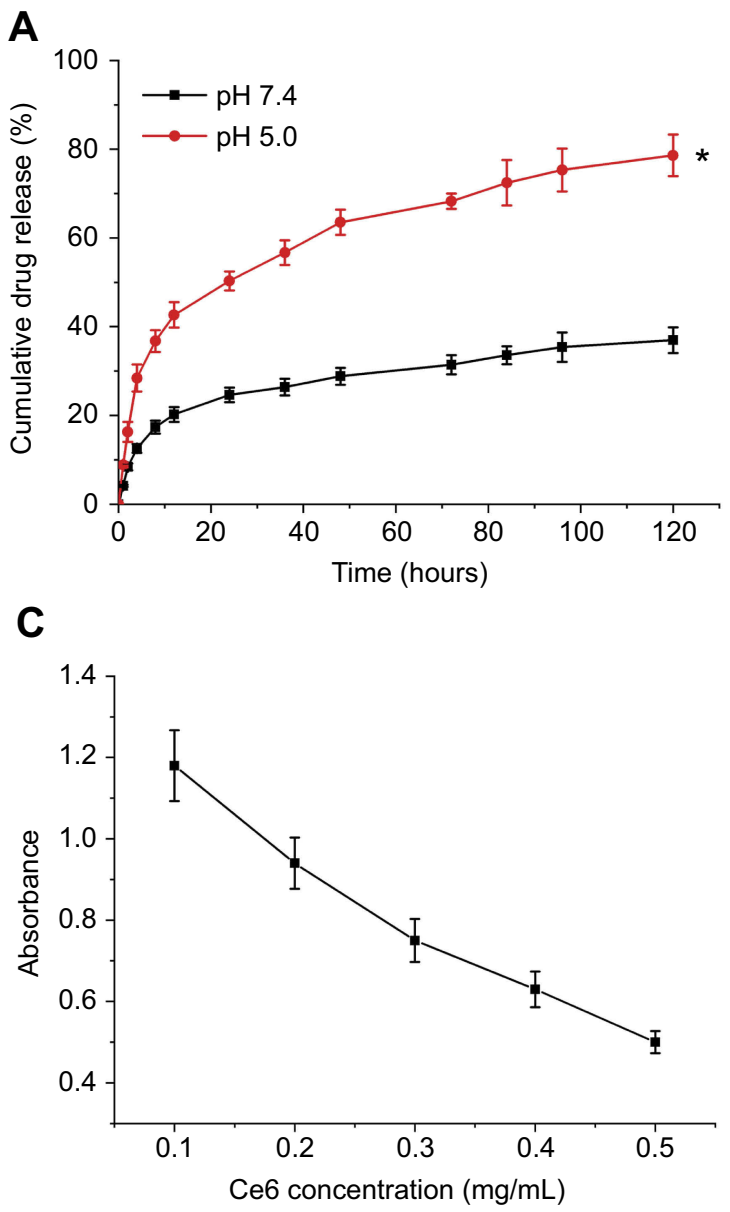

The preservation of $\mathrm{Ce} 6$ to generate ROS under laser irritation is a prerequisite for $\mathrm{CM} / \mathrm{SLN} / \mathrm{Ce} 6$ to exert PDT. To verify this, DPBF was employed to measure the the ROS generation capacity of CM/SLN/Ce6. It is generally recognized that the UV absorbance of DPBF at $418 \mathrm{~nm}$ would decrease when DPBF reacts with neighboring ROS. As a result, the variation of DPBF absorption at $418 \mathrm{~nm}$ was identified as an easy tool to evaluate the ROS generation of Ce6. As shown in Figure 3B, under laser irradiation $\left(1 \mathrm{~W} / \mathrm{cm}^{2}\right), \mathrm{UV}$ absorption at $415 \mathrm{~nm}$ showed a continuous decrease at constant $\mathrm{CM} / \mathrm{SLN} / \mathrm{Ce} 6$ concentration (Ce6: $0.1 \mathrm{mg} / \mathrm{mL}$ ) with $72 \%$ of absorption quench at 6 minutes post irradiation. The concentration dependent variations of DPBF absorption at $415 \mathrm{~nm}$ was also investigated. Various concentrations of $\mathrm{CM} / \mathrm{SLN} / \mathrm{Ce} 6$ (Ce6 concentration: $0.1-0.5 \mathrm{mg} / \mathrm{mL})$ were irradiated $1 \mathrm{~W} / \mathrm{cm}^{2}$ for 60 seconds

B
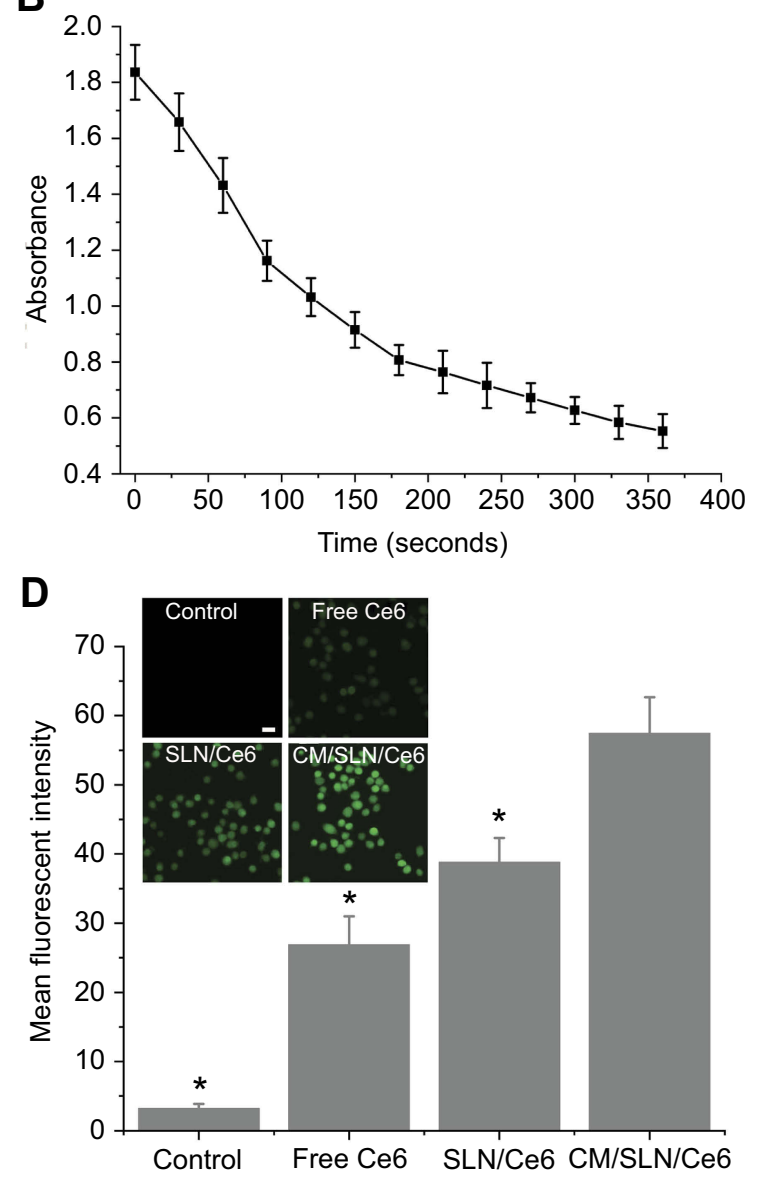

Figure 3 (A) Drug release profiles of Ce6 from the CM/SLN/Ce6 in release media under extracellular and intracellular condition of pHs (7.4 and 5.5). (B) Variation on absorbance

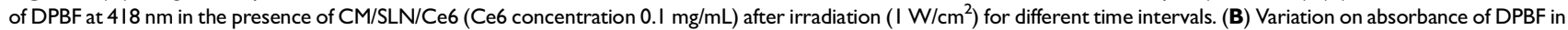
the presence of different concentrations of CM/SLN/Ce6 (Ce6 concentration: $0.1-0.5 \mathrm{mg} / \mathrm{mL}$ ) under irradiation for 60 seconds. (D) The in vitro ROS generation analysis of CM/ $\mathrm{SLN} / \mathrm{Ce}$ 6. Main figure illustrated flow cytometric analysis of MFI in cells treated by different formulations after 5 minutes of laser irradiation $\left(\mathrm{I} \mathrm{W} / \mathrm{cm}^{2}\right)$. Inset images demonstrated the representing CLSM images of cells treated with different formulations.

Notes: Significant differences were vs CM/SLN/Ce6. Scale bar: $20 \mu \mathrm{m}$. Data were shown as mean $\pm S D(N=3)$. ${ }^{*} p<0.05$.

Abbreviations: Ce6, chlorins e6; CM, cellular membranes; SLN, silica nanoparticles; DPBF, I,3-diphenylisobenzofuran; MFI, mean fluorescence intensity. 
and the UV absorption at $415 \mathrm{~nm}$ was recorded. As shown in Figure 3C, the ROS production of $\mathrm{CM} / \mathrm{SLN} / \mathrm{Ce} 6$ was positively related to the $\mathrm{Ce} 6$ concentration upon laser irradiation. Both results suggested that the encapsulated Ce6 within CM/SLN/Ce6 preserved the capability to generate ROS.

The intracellular ROS generation profile of CM/SLN/ Ce6 was further investigate using DCFH-DA. DCF, the oxidized product of DCFH-DA, shows green fluorescence which can be easily detected at Ex $485 \mathrm{~nm} / \mathrm{Em} 530 \mathrm{~nm}$ and can be quantified using flow cytometry. As shown in Figure 3D, after laser irradiation, no observable fluorescent signal was detected in cells without Ce6 (control group), which suggested that the ROS level in these cells was weak. Observable weak fluorescent signal was found in cells treated with free Ce6. In addition, significantly enhanced ROS concentration was detected in SLN/Ce6 treated cell, indicating that the intracellular Ce6 in this group might be higher than free Ce6 group. Most importantly, fluorescent intensity of DCF in cells treated with $\mathrm{CM} / \mathrm{SLN} / \mathrm{Ce} 6$ was much more potent than other groups, suggesting effective ROS production. CLSM (inset images) also confirmed these results. Therefore, CM/SLN/Ce6 with effective delivery efficacy was suggested to be a preferable DDS for PDT of cancer.

\section{In vitro anticancer assay}

The standard MTT assay was adopted in our study to investigate the in vitro anticancer efficiency of CM/SLN/ Ce6. The viability of cells exposed to drug free CM/SLN still over $90 \%$ at the highest concentration (Figure 4A), which suggested that $\mathrm{CM} / \mathrm{SLN}$ holds preferable biocompatibility with negligible cytotoxicity. The following cytotoxicity assay using drug loaded formulation was shown in Figure 4B. It was observed that under laser irradiation, CM/SLN/Ce6 showed superior anticancer efficacy than the other two groups at all tested concentrations. To further verify this conclusion, the apoptosis related proteins (cleaved caspase-3, bcl-2 and cytochrome-3) in three tested groups (Ce6 concentration: 5 $\mu \mathrm{g} / \mathrm{mL}$ ) were assessed. ${ }^{43-45}$ As shown in the inset images, as expected, the level of cleaved caspase-3 was higher in $\mathrm{CM} / \mathrm{SLN} / \mathrm{Ce} 6$ treated cells while bcl-2 (responsible for suppressing apoptosis) was much lower than other groups, which further confirming the preferable anticancer efficacy of $\mathrm{CM} / \mathrm{SLN} / \mathrm{Ce} 6$. In addition, the CM/SLN/ Ce6 showed much higher level of cytochrome-3, which indicated that mitochondria damage was also involved in the apoptosis of cells. ${ }^{46}$

\section{Cellular uptake assay of $\mathrm{cm} / \mathrm{s} / \mathrm{n} / \mathrm{ce} 6$}

In order to reveal the potential reasons responsible for the discriminative anticancer efficacy of different formulations, in vitro cellular uptake assay was performed to investigate if SGC7901 CM modification could increase the internalization of CM/SLN/Ce6 to SGC7901 cells.

As displayed in Figure 5A, the increase of fluorescentsignal positively related to the incubation time in both groups. However, free Ce6 showed the most inferior intracellular accumulation compared to both nanoparticles,
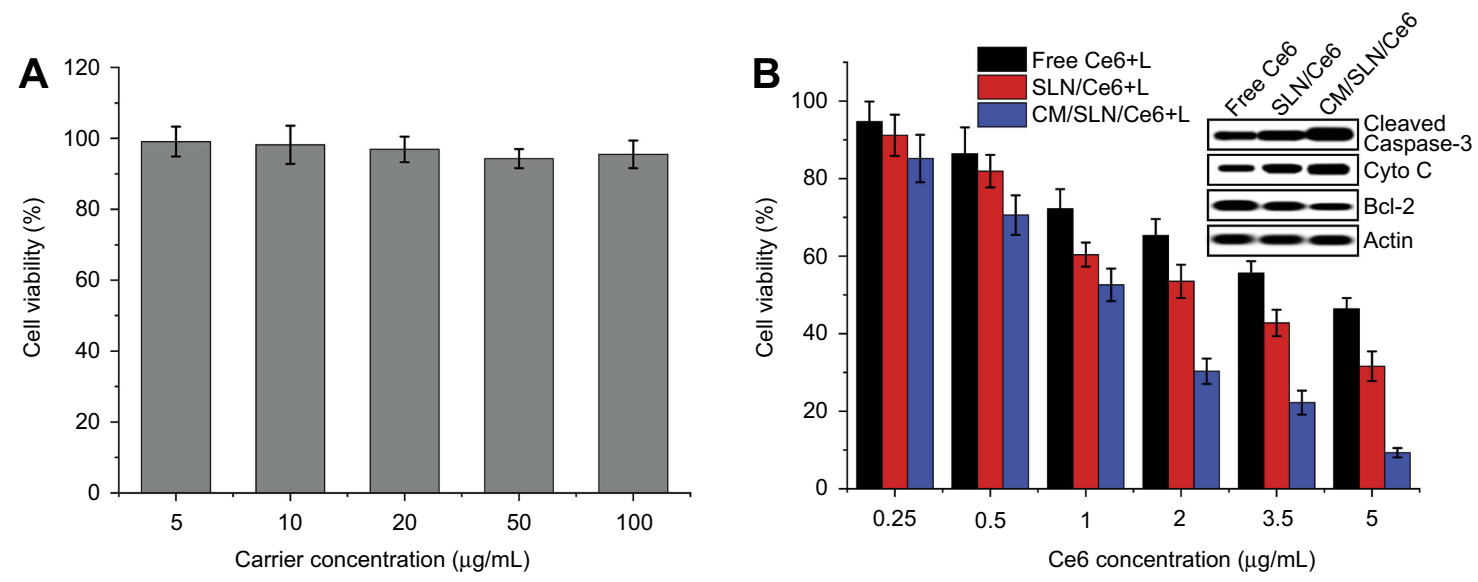

Figure 4 (A) Cytotoxicity of CM/SLN after 48 hours incubation with SGC790I cells. (B) Cytotoxicity of free Ce6, SLN/Ce6 and CM/SLN/Ce6 (with laser irradiation) at different Ce6 concentrations against SGC790I cells after 48 hours incubation. Inset images demonstrated the cleaved caspase-3, cytochrome C and bcl-2 levels in three groups (Ce6 concentration: $5 \mu \mathrm{g} / \mathrm{mL}$ ).

Note: Data were shown as mean $\pm \mathrm{SD}(\mathrm{N}=3)$.

Abbreviations: Ce6, chlorins e6; CM, cellular membranes; SLN, silica nanoparticles. 
A

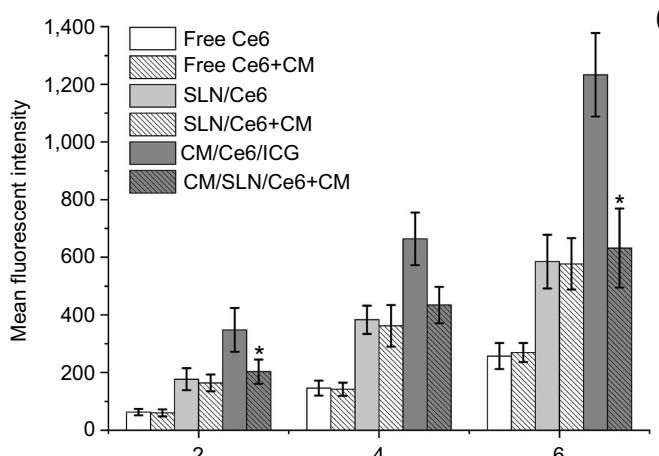

B

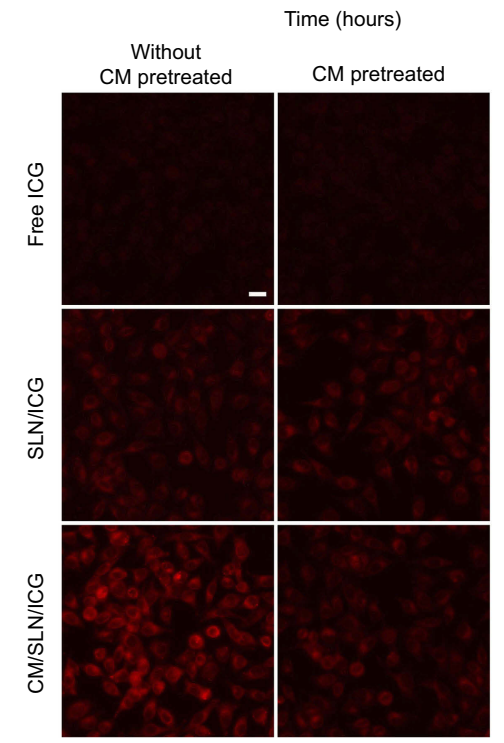

C

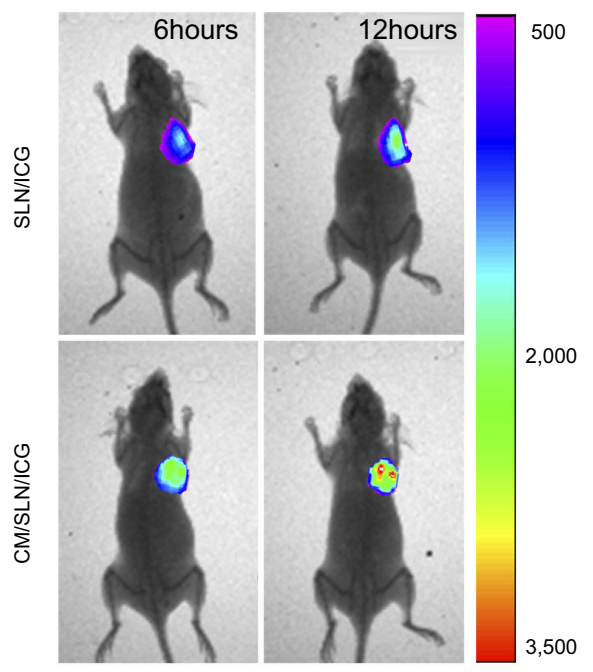

D

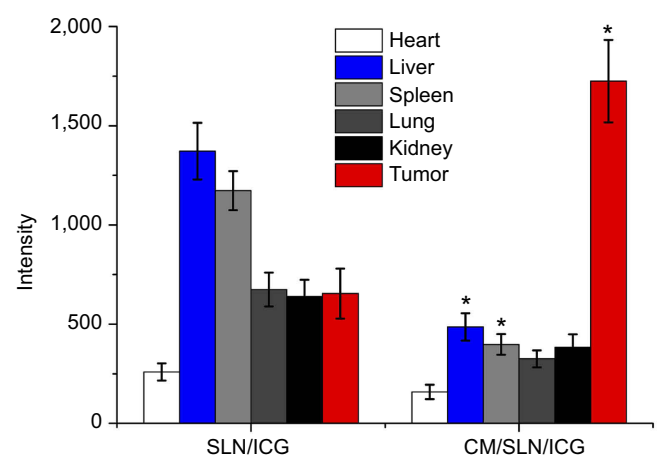

Figure 5 (A) Quantitative analysis of intracellular time-dependent uptake of different formulations in SGC790I cells (pretreated with/without CM). Significant differences were vs groups pretreated without CM. (B) Representative CLSM images of uptake of different formulations in SGC790I cells (pretreated with/without CM) at 4 hours postincubation. Scale bar: $20 \mu \mathrm{m}$. (C) In vivo real time distribution of SLN/ICG and CM/SLN/ICG at 6 and 12 hours postincubation. (D) Mean fluorescentintensity of dissected tumors and major organs of mice treated with SLN/ICG and CM/SLN/ICG at 48 hours postinjection.

Note: Data were expressed as mean $\pm \mathrm{SD}(\mathrm{N}=3)$.

Abbreviations: Ce6, chlorins e6; CM, cellular membranes; SLN, silica nanoparticles; CLSM, confocal laser scanning microscope; ICG, indocyanine green dye.

which was inconsistent with a previous report that nanoparticles can improve the uptake of drugs into cells. ${ }^{35}$ In addition, it was observed that higher $\mathrm{Ce} 6$ signals were observed in CM/SLN/Ce6 group than SLN/Ce6 under all tested conditions. In order to reveal the role of $\mathrm{CM}$ in cellular uptake, cells were pretreated with excess $\mathrm{CM}$ prior to addition of nanoparticles. Interestingly, it was observed that the cellular $\mathrm{Ce} 6$ intensity in $\mathrm{CM} / \mathrm{SLN} / \mathrm{Ce} 6$ group suffered a significant decline while SLN/Ce6 group showed insignificant variations, which was also verified by CLSM imaging of cells at 4 hours postincubation (Figure 5B). These results suggested that the internalization of $\mathrm{CM} / \mathrm{SLN} / \mathrm{Ce} 6$ into SGC7901 cells was positively related to $\mathrm{CM}$ modification, possibly via $\mathrm{CM}$ mediated endocytosis.

\section{In vivo distribution}

SGC7901 CM modification was expected to assist the tumor-homing of $\mathrm{CM} / \mathrm{SLN} / \mathrm{ICG}$ to the isogenous SGC7901 cells to increasing the accumulation of nanoparticles in the tumor tissue. In order to verify the hypothesis, the real time distribution of SLN/ICG and CM/SLN/ICG was monitored by in vivo imaging system at 6 and 12 hours postadministration. As shown in Figure $5 \mathrm{C}$, as expected, CM/SLN/ICG showed much effective accumulation within the tumor tissue compared to SLN/ICG at both time intervals. In addition, the fluorescent distribution of tumor and major organs obtained by ex vivo imaging also confirmed this observation. As shown in Figure 5D, due to the poor tumor targetability of SLN/ICG, the ICG signal in this group was shown to be largely located in the 
A

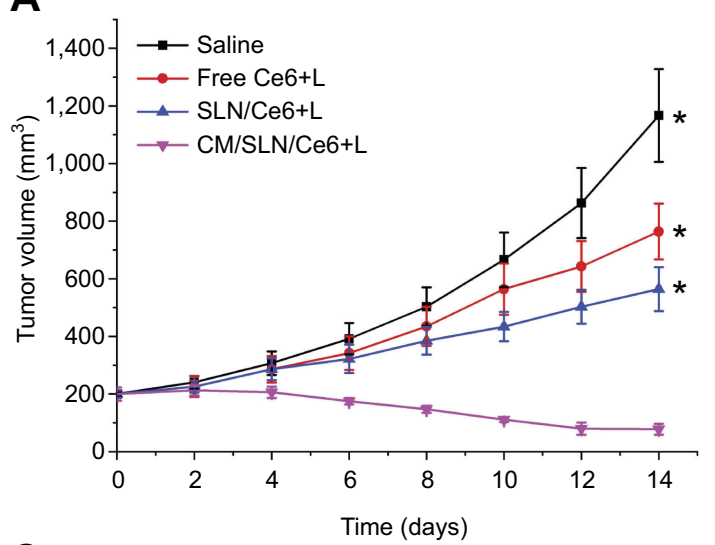

C
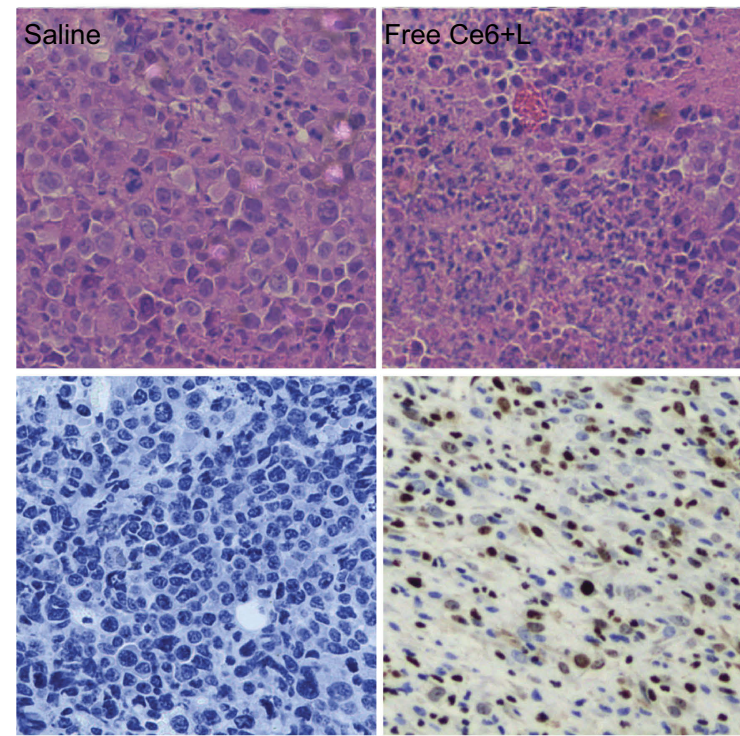

B
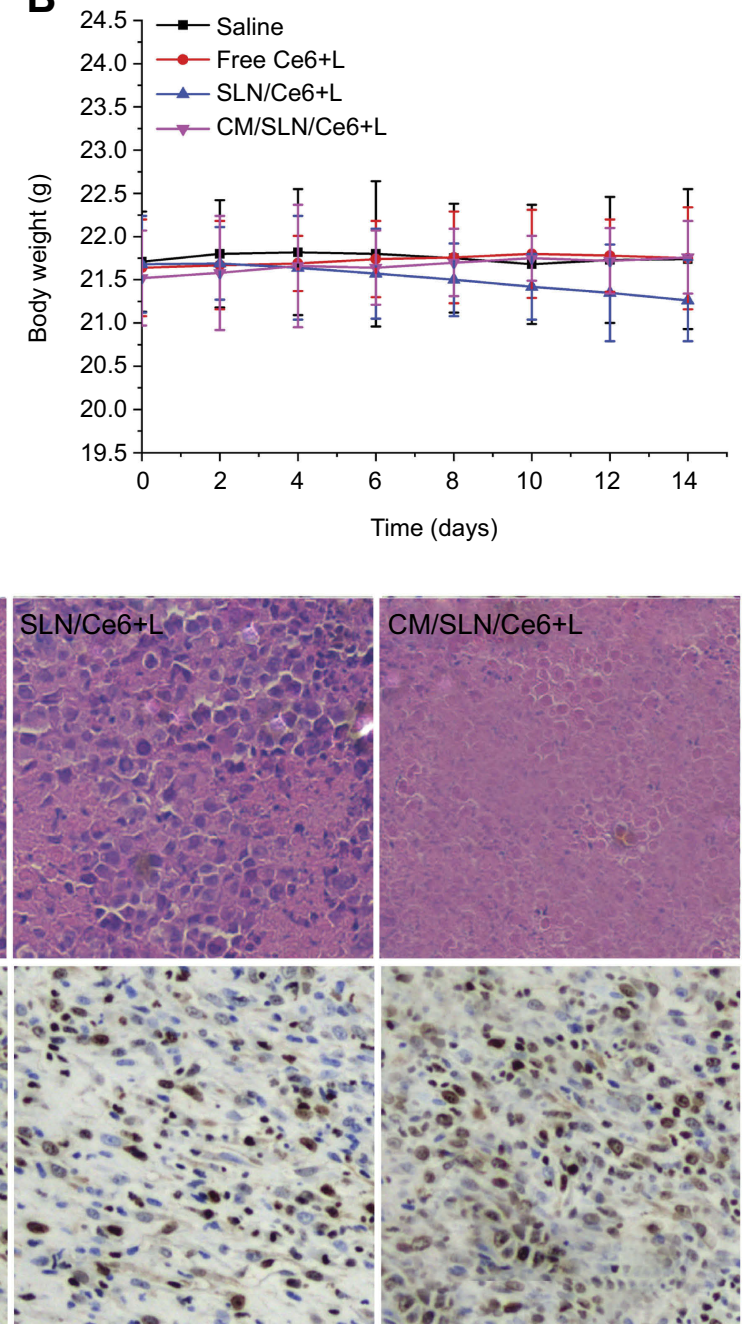

CM/SLN/Ce6+L

Figure 6 The tumor volume (A), body weight (B) and H\&E/TUNEL staining (200x) of tumor tissue (C) analysis of SGC790I tumor-bearing Balb/c nude mice after intravenous administration of different formulations.

Notes: Data were expressed as mean $\pm S D(N=6)$. Significant differences were vs CM/SLN/Ce6. ${ }^{*} p<0.05$.

Abbreviations: Ce6, chlorins e6; CM, cellular membranes; SLN, silica nanoparticles.

liver and kidneys. In contrast, the distribution profile of $\mathrm{CM} / \mathrm{SLN} / \mathrm{ICG}$ is significantly changed with less accumulation in liver and kidney while enhanced homing to tumor tissue.

\section{In vivo anticancer assay}

In vivo anticancer efficacy of $\mathrm{CM} / \mathrm{SLN} / \mathrm{Ce} 6$ was assessed. The tumor growth profiles of different formulations were displayed in Figure 6A. It was observed that free $\mathrm{Ce} 6$ or SLN/Ce6 could exert certain suppression effects on tumor growth. By contrast, the anticancer effect of $\mathrm{CM} / \mathrm{SLN} / \mathrm{Ce} 6$ was much more potent than the others with a final tumor volume of $78 \pm 19 \mathrm{~mm}^{3}$ at the end of the test. Moreover, body weight variations of mice in different groups also revealed some interesting results. As shown in Figure 6B, no significant decline in body weight was observed in $\mathrm{CM} / \mathrm{SLN} / \mathrm{Ce} 6$ group, suggesting that the preferable tumor homing of $\mathrm{CM} / \mathrm{SLN} / \mathrm{Ce} 6$ could increase its anticancer efficacy while reduce the undesired toxic effects. In contrast, the untargeted distribution of SLN/Ce6 caused systematic toxicity to mice which was reflected by the timedependent decrease in body weight. Moreover, H\&E and TUNEL assays were conducted to assess the apoptosis profile of tumor tissues treated by different formulations (Figure 6C). Compared to the insignificant apoptosis in saline group, increased apoptosis was observed in all $\mathrm{Ce} 6$ containing groups with the most serious one observed in $\mathrm{CM} / \mathrm{SLN} / \mathrm{Ce} 6$ group, which was in line with results obtained in the above assays. In a word, the $\mathrm{CM} / \mathrm{SLN} / \mathrm{Ce} 6$ holds great potential to be a preferable tumor-targeting DDS for effective PDT of gastric cancer. 


\section{Conclusion}

In our study, we successfully fabricated a Ce6 loaded and tumor-targeted DDS using SGC7901 CM modified SLN (CM/SLN/Ce6). In this platform, we combined the tumortargeting nature of $\mathrm{CM}$ and the decent loading capacity of SLN. Experimental results revealed that $\mathrm{CM} / \mathrm{SLN} / \mathrm{Ce} 6$ was able to specifically deliver $\mathrm{Ce} 6$ to isogenous SGC7901 cells. Furthermore, the CM/SLN/Ce6 was capable of releasing the loaded drug in a $\mathrm{pH}$ dependent manner. It was noted that both in vitro and in vivo experiments revealed that $\mathrm{CM} / \mathrm{SLN} / \mathrm{Ce} 6$ hold superior tumortargeting ability with greatly enhanced anticancer efficacy than unmodified SLN and free drug.

\section{Disclosure}

The authors report no conflicts of interest in this work.

\section{References}

1. Miron RJ, Zhang Y. Autologous liquid platelet rich fibrin: a novel drug delivery system. Acta Biomater. 2018;75:35-51. doi:10.1016/j. actbio.2018.05.021

2. Xie J, Ji Y, Xue W, Ma D, Hu Y. Hyaluronic acid-containing ethosomes as a potential carrier for transdermal drug delivery. Coll Surf B. 2018;172:323-329. doi:10.1016/j.colsurfb.2018.08.061

3. Zhao L, Xiao C, Wang L, Gai G, Ding J. Glucose-sensitive polymer nanoparticles for self-regulated drug delivery. Chem Commun. 2016;52(49):7633-7652. doi:10.1039/c6cc02202b

4. Zhou M, Shen L, Lin X, Hong Y, Feng Y. Design and pharmaceutical applications of porous particles. RSC Adv. 2017;7(63):39490-39501. doi:10.1039/C7RA06829H

5. Rainone P, Riva B, Belloli S, et al. Development of $99 \mathrm{mTc}$-radiolabeled nanosilica for targeted detection of HER2-positive breast cancer. Int J Nanomed. 2017;12:3447-3461. doi:10.2147/IJN.S129720

6. Tang D, Zhao X, Yang T, Wang C. Paclitaxel prodrug based mixed micelles for tumor-targeted chemotherapy. RSC Adv. 2018;8 (1):380-389. doi:10.1039/C7RA07796C

7. Meng L-X, Ren Q, Meng Q, et al. Trastuzumab modified silica nanoparticles loaded with doxorubicin for targeted and synergic therapy of breast cancer. Artif Cells Nanomed Biotechnol. 2018;46:1-8.

8. Xiong H, Du S, Zhang P, Jiang Z, Zhou J, Yao J. Primary tumor and pre-metastatic niches co-targeting "peptides-lego" hybrid hydroxyapatite nanoparticles for metastatic breast cancer treatment. Biomat Sci. 2018;6(10):2591-2604. doi:10.1039/c8bm00706c

9. Daryasari MP, Akhgar MR, Mamashli F, Bigdeli B, Khoobi M. Chitosan-folate coated mesoporous silica nanoparticles as a smart and $\mathrm{pH}$-sensitive system for curcumin delivery. Rsc Adv. 2016;6 (107):105578-105588. doi:10.1039/C6RA23182A

10. Martínezcarmona M, Lozano D, Colilla M, Valletregí M. Selective topotecan delivery to cancer cells by targeted $\mathrm{pH}$-sensitive mesoporous silica nanoparticles. Rsc $A d v$. 2016;6(56):50923-50932. doi:10.1039/C6RA07763C

11. Chu Y, Chen N, Yu H, et al. Topical ocular delivery to laser-induced choroidal neovascularization by dual internalizing RGD and TAT peptide-modified nanoparticles. Int J Nanomed. 2017;12:1353-1368. doi:10.2147/IJN.S126865
12. Liu C-M, Chen G-B, Chen -H-H, et al. Cancer cell membrane-cloaked mesoporous silica nanoparticles with a $\mathrm{pH}$-sensitive gatekeeper for cancer treatment. Coll Surf B. 2019;175:477-486. doi:10.1016/j. colsurfb.2018.12.038

13. Martínez-Carmona M, Lozano D, Colilla M, Vallet-Regí M. Lectinconjugated $\mathrm{pH}$-responsive mesoporous silica nanoparticles for targeted bone cancer treatment. Acta Biomater. 2018;65:393-404. doi:10.1016/j.actbio.2017.11.007

14. Wang C, Wang Z, Zhao X, et al. DOX loaded aggregation-induced emission active polymeric nanoparticles as a fluorescence resonance energy transfer traceable drug delivery system for self-indicating cancer therapy. Acta Biomater. 2019;85:218-228. doi:10.1016/j. actbio.2018.12.020

15. Xiong H, Ni J, Jiang Z, Tian F, Zhou J, Yao J. Intracellular self-disassemble polysaccharide nanoassembly for multi-factors tumor drug resistance modulation of doxorubicin. Biomat Sci. 2018;6(9):2527-2540. doi:10.1039/c8bm00570b

16. Wang C, Han M, Liu X, et al. Mitoxantrone-preloaded water-responsive phospholipid-amorphous calcium carbonate hybrid nanoparticles for targeted and effective cancer therapy. Int J Nanomed. 2019;14:1503-1517. doi:10.2147/IJN.S193976

17. Kumar Mehata A, Bharti S, Singh P, et al. Trastuzumab decorated TPGS-g-chitosan nanoparticles for targeted breast cancer therapy. Coll Surf B. 2019;173:366-377. doi:10.1016/j.colsurfb.2018.10.007

18. Kennedy PJ, Sousa F, Ferreira D, et al. Fab-conjugated PLGA nanoparticles effectively target cancer cells expressing human CD44v6. Acta Biomater. 2018;81:208-218. doi:10.1016/j.actbio.2018.09.043

19. Yan L, Wang Z, Chen X, et al. Firmly anchored photosensitizer Chlorin e6 to layered double hydroxide nanoflakes for highly efficient photodynamic therapy in vivo. Chem Commun. 2017;53 (15):2339-2342. doi:10.1039/c6cc09510k

20. Luo L, Zhong H, Liu S, et al. Intracellular "activated" two-photon photodynamic therapy by fluorescent conveyor and photosensitizer co-encapsulating $\mathrm{pH}$-responsive micelles against breast cancer. Int J Nanomed. 2017;12(12):5189-5201. doi:10.2147/IJN.S140345

21. Zhang C, Cheng X, Chen M, et al. Fluorescence guided photother$\mathrm{mal} /$ photodynamic ablation of tumours using $\mathrm{pH}$-responsive chlorin e6-conjugated gold nanorods. Coll Surf B. 2017;160:345-354. doi:10.1016/j.colsurfb.2017.09.045

22. Zhou X, Chen Y, Su J, Tian X, Luo Y, Luo L. In situ second-harmonic generation mediated photodynamic therapy by micelles co-encapsulating coordination nanoparticle and photosensitizer. RSC Adv. 2017;7(82):52125-52132. doi:10.1039/ C7RA07334H

23. Xiang SD, Wilson KL, Goubier A, Heyerick A, Plebanski M. Design of Peptide-Based Nanovaccines Targeting Leading Antigens From Gynecological Cancers to Induce HLA-A2.1 Restricted CD8(+) T Cell Responses. Front Immunol. 2018;9:2968. doi:10.3389/fimmu.2018.02968

24. Li X, Zhao X, Pardhi D, et al. Folic acid modified cell membrane capsules encapsulating doxorubicin and indocyanine green for highly effective combinational therapy in vivo. Acta Biomater. 2018;74:374-384. doi:10.1016/j.actbio.2018.05.006

25. Kilic A, Kok FN. Peptide-functionalized supported lipid bilayers to construct cell membrane mimicking interfaces. Coll Surf $B$. 2019;176:18-26. doi:10.1016/j.colsurfb.2018.12.052

26. Hu CMJ, Li Z, Santosh A, Connie C, Fang RH, Liangfang ZJ. Erythrocyte membrane-camouflaged polymeric nanoparticles as a biomimetic delivery platform. Proc Nat Acad Sci U.S.A. 2011;108 (27):10980-10985. doi:10.1073/pnas.1106634108

27. Gao C, Lin Z, Juradosánchez B, Lin X, Wu Z, He QJS. Stem cell membrane-coated nanogels for highly efficient in vivo tumor targeted drug delivery. Small. 2016;12(30):4056-4062. doi:10.1002/ smll.201600624

28. Yurkin ST, Wang ZJN. Cell membrane-derived nanoparticles: emerging clinical opportunities for targeted drug delivery. Nanomedicine. 2017;12(16):2007-2019. doi:10.2217/nnm-2017-0100 
29. Zhao X, Tang D, Yang T, Wang C. Facile preparation of biocompatible nanostructured lipid carrier with ultra-small size as a tumor-penetration delivery system. Coll Surf B. 2018;170:355-363. doi:10.1016/j.colsurfb.2018.06.017

30. Gu L, Shi T, Sun Y, et al. Folate-modified, indocyanine green-loaded lipid-polymer hybrid nanoparticles for targeted delivery of cisplatin. J Biomater Sci Polym Ed. 2017;28(7):690-702. doi:10.1080/ 09205063.2017.1296347

31. Suski JM, Magdalena L, Aleksandra W, et al. Isolation of plasma membrane-associated membranes from rat liver. Nat Protoc. 2014;9 (2):312-322. doi:10.1038/nprot.2014.016

32. Yurkin ST, Wang Z. Cell membrane-derived nanoparticles: emerging clinical opportunities for targeted drug delivery. Nanomedicine. 2017;12(16):2007-2019. doi:10.2217/nnm-2017-0100

33. Wang C, Chen S, Wang Y, et al. Lipase-triggered water-responsive "pandora's box" for cancer therapy: toward induced neighboring effect and enhanced drug penetration. Adv Mater. 2018;30 (14):1706407. doi:10.1002/adma.201706407

34. Ding X, Xu X, Zhao Y, et al. Tumor targeted nanostructured lipid carrier co-delivering paclitaxel and indocyanine green for laser triggered synergetic therapy of cancer. Rsc Adv. 2017;7 (56):35086-35095. doi:10.1039/C7RA06119F

35. Wang Y, Wang C, Ding Y, et al. Biomimetic HDL nanoparticle mediated tumor targeted delivery of indocyanine green for enhanced photodynamic therapy. Coll Surf B. 2016;148:533-540. doi:10.1016/ j.colsurfb.2016.09.037

36. Zhang X, Tan X, Zhang D, et al. A cancer cell specific targeting nanocomplex for combination of mRNA-responsive photodynamic and chemo-therapy. Chem Commun. 2017;53(72):9979-9982. doi:10.1039/c7cc05295b

37. Tang D, Zhao X, Zhang L, Wang Z, Wang C. Identification of hub genes to regulate breast cancer metastasis to brain by bioinformatics analyses. J Cell Biochem. 2019;120(6):9522-9531. doi:10.1002/jcb.28228
38. Wu L, Ni C, Zhang L, et al. Surface charge convertible and biodegradable synthetic zwitterionic nanoparticles for enhancing cellular drug uptake. Macromol Biosci. 2016;16(3):308-313. doi:10.1002/ mabi.201500299

39. Fang $\mathrm{R}, \mathrm{Hu} \mathrm{CMJ}$, Luk BT, et al. Cancer cell membrane-coated nanoparticles for anticancervaccination and drug delivery. Nano Lett. 2014;14(4):2181-2188. doi:10.1021/n1500618u

40. Tang D, Zhao X, Zhang L, Wang Z, Wang C. Identification of hub genes to regulate breast cancer metastasis to brain by bioinformatics analyses. J Cel Biochem. 2019;120:9522-9531.

41. Hashemi M, Yadegari A, Yazdanpanah G, Jabbehdari S, Omidi M, Tayebi L. Functionalized R9-reduced graphene oxide as an efficient nano-carrier for hydrophobic drug delivery. RSC Advances. 2016;6 (78):74072-74084. doi:10.1039/C6RA13822E

42. Sheng Y, Chang L, Kuang T, Hu J. PEG/heparin-decorated lipidpolymer hybrid nanoparticles for long-circulating drug delivery. Rsc Adv. 2016;6(28):23279-23287. doi:10.1039/C5RA26215A

43. Yang J, Hu S, Rao M, et al. Copper nanoparticle-induced ovarian injury, follicular atresia, apoptosis, and gene expression alterations in female rats. Int J Nanomed. 2017;12:5959-5971. doi:10.2147/IJN. S139215

44. Zhang L, Hao $\mathrm{W}, \mathrm{Xu} \mathrm{L}$, et al. A pH-sensitive methenamine mandelate-loaded nanoparticle induces DNA damage and apoptosis of cancer cells. Acta Biomater. 2017;62:246-256. doi:10.1016/j. actbio.2017.08.019

45. Zhang -T-T, Yang F, Li X-L, Zhao W, Xu -J-J, Chen H-Y A multifunctional silver nanocomposite for the apoptosis of cancer cells and intracellular imaging. Chem Commun. 2017;53 (41):5614-5617. doi:10.1039/c7cc02834b

46. Cheng TN, Liang QY, Hande MP, et al. Zinc oxide nanoparticles exhibit cytotoxicity and genotoxicity through oxidative stress responses in human lung fibroblasts andDrosophila melanogaster. Int J Nanomed. 2017;12:1621-1637. doi:10.2147/IJN.S124403
International Journal of Nanomedicine

\section{Publish your work in this journal}

The International Journal of Nanomedicine is an international, peerreviewed journal focusing on the application of nanotechnology in diagnostics, therapeutics, and drug delivery systems throughout the biomedical field. This journal is indexed on PubMed Central, MedLine, CAS, SciSearch ${ }^{\mathbb{R}}$, Current Contents ${ }^{\mathbb{B}} /$ Clinical Medicine, $^{2}$
Journal Citation Reports/Science Edition, EMBase, Scopus and the Elsevier Bibliographic databases. The manuscript management system is completely online and includes a very quick and fair peer-review system, which is all easy to use. Visit http://www.dovepress.com/ testimonials.php to read real quotes from published authors. 